\title{
The Mental Health Care Context and Patient Characteristics: Implications for Provider Job Satisfaction
}

\author{
C. Raymond Bingham, PhD \\ Marcia Valenstein, MD \\ Frederic C. Blow, PhD \\ Jeffrey A. Alexander, PhD
}

\begin{abstract}
This research examines job satisfaction among 282 staff providing mental health care to 574 patients with serious mental illness. The mental health staff worked in 18 Department of Veterans Affairs inpatient and outpatient mental health care units at 12 Veterans Affairs Medical Centers located across the contiguous 48 states. The purpose was to identify (1) aspects of the health care context that were associated with provider job satisfaction and (2) administrative and organizational procedures/interventions that might sustain or improve provider job satisfaction. The association of provider job satisfaction with patients' functional status and symptom severity was tested in multilevel statistical models that accounted for provider and unit characteristics. Provider job satisfaction was found to be greater on smaller units and units with higher patient functioning and lower illness severity. Implications of these results are discussed.
\end{abstract}

\section{Introduction}

Low levels of job satisfaction and high rates of staff burnout and turnover are common among providers serving challenging patient populations, such as patients with serious mental illness (SMI). ${ }^{1-19}$ This research used unit-level aggregate measures of patient functional status and symptom severity to identify patient characteristics associated with low provider job satisfaction. The associations between job satisfaction, burnout, and turnover among health care providers suggest that managerial and policy interventions that enhance job satisfaction also may reduce stress, burnout, and turnover of health care providers. ${ }^{20-26}$ A better understanding of the role of patient characteristics

Address correspondence to $\mathrm{C}$. Raymond Bingham, $\mathrm{PhD}$, Research Investigator. Department of Veterans Affairs, and Assistant Research Scientist. Department of Psychiatry, University of Michigan. HSR\&D, PO Box 130170, Ann Arbor, MI 48113-0170; e-mail: rbingham@umich.edu.

Marcia Valenstein, MD, is a Research Investigator. Department of Veterans Affairs, and Assistant Clinical Professor II, Department of Psychiatry, University of Michigan.

Frederic C. Blow, PhD, is Director, Serions Mental Illness Treatment Research and Evaluation Center. Department of Veterans Affairs, and Associate Professor and Senior Associate Research Scientist, Department of Psychiatry. University of Michigan.

Jeffrey A. Alexander, PhD, is a Research Investigator, Department of Veterans Affairs, and Professor. Health Management and Policy, University of Michigan.

Journal of Behavioral Health Services \& Research, 2002, 29(3), 335-344. This article was created in the public domain. 
in these associations will help to improve the effectiveness of efforts to increase retention, while reducing the loss of skilled health care providers.

Provider job satisfaction is influenced by various workplace characteristics, including size, type, and location (ie, medical center, inpatient or outpatient clinic), and administrative organization. The health care workplace also is shaped by the collection of symptoms, needs, and conditions of the patients receiving care. Collectively, patients' health attributes influence the array of health care procedures employed; limit the range of expectable patient outcomes; contribute to the type, quantity, and quality of interaction between patients and providers; and impact rewards and difficulties encountered in providing health care.

This research examined the patient contribution to the health care context and its association with health care providers' job satisfaction. The patient contribution to the health care context was measured by aggregating survey and clinical data collected from patients receiving care on the same inpatient or outpatient unit to form unit-level indexes of patients' symptomatic and functional levels. These indexes of unit-level patient characteristics were included in multilevel models to test the association between characteristics of the health care context (ie, unit and patient characteristics) and provider characteristics (ie, demographic and professional) and provider job satisfaction. Data collected directly from patients have not typically been used to characterize patient groups (ie, all patients

on a single unit). ${ }^{27}$ Instead, administrative data and surveys of health care supervisors and administrators have usually assessed the aggregate contribution of patients' characteristics to the health care context and to control for variation across units of analysis (ie, hospitals or clinic types). ${ }^{21,27-33}$ This research tested the contribution of patient characteristics (level-2, unit-level covariates) to providers' job satisfaction (level-1 outcome) using a two-level model. This modeling procedure controlled for variation across and within units in providers' and patients' characteristics (level-1, provider-level covariates). It is hoped the results will inform health care administrators and policy makers who wish to improve provider job satisfaction, reduce burnout, and decrease rates of turnover.

The study goals were to identify: (1) aspects of the health care context that are associated with provider job satisfaction and (2) administrative and organizational procedures/interventions that might sustain or improve provider job satisfaction. It was hypothesized that greater provider job satisfaction would be associated with lower patient illness severity and higher functioning. In addition, associations between specific characteristics of patients with SMI (ie, symptom severity and functional status) and provider job satisfaction were explored.

\section{Methods}

\section{Samples and data}

The data for this study were gathered from two groups of participants: patients receiving mental health care in the Department of Veterans Affairs (VA) health care system and the staff who provided their care. Data were gathered from providers who completed a survey of organizational and administrative factors relating to job satisfaction that was administered in fall 1992. Patients were participants in a study of VA mental health care programs for veterans with SMI and were enrolled into the study on a rolling basis from 1991 through 1995.

Assessment date and unit linked the two sets of data. Data for all patients who were surveyed within 12 months of the administration of the provider job satisfaction surveys were included in the study. The 12-month interval provided a representation of patients on the units when provider job satisfaction was assessed and yielded a large enough sample of units and providers to test the hypotheses of this study. The patient data were aggregated within unit to form unit-level indicators of patient functioning and illness severity. Data from the health care context (ie, unit and patient data) were linked to individual provider data so that their association with provider job satisfaction could be tested while controlling for individual provider characteristics. 
The provider and patient data were gathered from 18 units in 12 VA Medical Centers (VAMCs) located in Alabama, Colorado, Iowa, Kansas, Massachusetts, Maryland, Minnesota, New Jersey, New York, Pennsylvania, Tennessee, and Wyoming. At the time of data collection the VAMCs ranged in size from 189 to 983 beds. Three VAMCs were located in urban areas, while the remaining nine were in medium- to small-sized metropolitan areas and rural locations.

\section{Provider sample}

The provider sample $(n=282)^{34}$ included psychiatrists $(5.4 \%)$; psychologists $(4.8 \%)$; social workers $(12.4 \%)$; registered $(24.8 \%)$ and licensed practical nurses $(4.8 \%)$; nurse's aides $(26.3 \%)$; occupational $(4.1 \%)$, recreational $(6.0 \%)$, and physical therapists $(1.3 \%)$; dietitians $(0.6 \%)$; chaplains $(0.6 \%)$; clinical pharmacists $(0.6 \%)$; and various other providers $(7.6 \%)$. The single largest group of providers was nurses (registered and licensed practical nurses and nurse's aides), which totaled $55.9 \%$ of the sample. The second largest group was clinical mental health care providers (psychiatrists, psychologists, social workers), which composed $22.6 \%$ of the study sample. Providers were $80.6 \%$ white and $13.2 \%$ African American; $66.0 \%$ were married, $18.2 \%$ separated or divorced, $1.3 \%$ widowed, and $14.5 \%$ never married. Provider surveys were completed between August 8 and December 12, 1992 , with a response rate exceeding $98 \%$. Table 1 summarizes provider characteristics.

\section{Patient sample}

This study utilized data collected from 574 veterans receiving mental health care on the same 18 units and 12 VAMCs in which the providers worked. Inclusion criteria in the original study were: (1) eligible for VA hospital care; (2) Diagnostic and Statistical Manual of Mental Disorders, third edition, revised (DSM-III-R) diagnosis of a mental illness involving psychotic symptoms (ie, schizophrenia/schizo-affective disorder, bipolar disorder, major depression with psychosis, organic psychosis); and (3) 150 or more documented days of hospitalization or five or more inpatient admissions to any VAMC during the year prior to the survey.

\section{Measures}

\section{Provider measures}

The survey assessed nine multi-item dimensions of provider job satisfaction measured on a 7-point agree-disagree continuum. Scales were scored by calculating the mean item score. The items were patterned after instruments used previously to assess the job satisfaction of health care providers, but were modified, based on information from site visits and focused group discussions with health care providers, to be more appropriate for mental health care. The job satisfaction survey was pre-tested with mental health providers at a VA acute care hospital, and piloted at four VA psychiatric hospitals. The scales demonstrated acceptable discriminant validity. Internal consistency ranged from $\alpha=.60$ to $\alpha=.89 .{ }^{34}$ Global Job Satisfaction (four-item scale), Satisfaction with Workload (seven-item scale), and Satisfaction with Relationships with Patients (four-item scale) were examined in this research.

Provider demographics, job tenure, and occupation were assessed in the provider survey by singleitem measures. Provider characteristics and job satisfaction are summarized in Table 1.

\section{Unit characteristics}

Two unit characteristics were examined in this study: unit type and unit size. Unit type was a dichotomous indicator with $1=$ inpatient unit and $0=$ outpatient unit. Unit size was calculated as the number of staff assigned to work on each unit. Although workload was not measured in this study, it was assumed that the ratio of patients to staff was relatively stable across units of similar type (ie, inpatient versus outpatient). 
Table 1

Provider and patient characteristics by unit type $(n=282)$

\begin{tabular}{|c|c|c|c|}
\hline & $\begin{array}{c}\text { Outpatient } \\
\text { Mean (SD) } \\
(n=66)\end{array}$ & $\begin{array}{c}\text { Inpatient } \\
\text { Mean (SD) } \\
(n=216)\end{array}$ & $p>|t|^{\dagger}$ \\
\hline \multicolumn{4}{|l|}{ Provider characteristics } \\
\hline Age & $42.7(9.0)$ & $43.1(9.7)$ & .758 \\
\hline Gender* & $51.5 \%$ & $64.8 \%$ & .053 \\
\hline Education & $16.2(1.2)$ & $14.8(2.0)$ & .000 \\
\hline Professional tenure & $3.6(1.4)$ & $3.6(1.3)$ & .816 \\
\hline VA tenure & $2.8(1.1)$ & $2.9(1.1)$ & .358 \\
\hline Position tenure & $1.8(1.0)$ & $2.1(1.1)$ & .032 \\
\hline Occupational rank & $3.2(1.4)$ & $2.7(1.2)$ & .003 \\
\hline \multicolumn{4}{|l|}{ Job satisfaction } \\
\hline Global & $5.4(1.2)$ & $4.9(1.5)$ & .020 \\
\hline Relationships with patients & $5.7(0.8)$ & $5.2(1.0)$ & .000 \\
\hline Workload & $3.8(1.3)$ & $3.9(1.3)$ & .434 \\
\hline \multicolumn{4}{|l|}{ Patient characteristics } \\
\hline \multicolumn{4}{|l|}{ Patient functioning } \\
\hline ADLs & $0.2(0.3)$ & $0.4(0.5)$ & .000 \\
\hline IADLs & $0.9(0.6)$ & $1.3(0.8)$ & .000 \\
\hline Social contact & $1.3(0.9)$ & $0.8(0.8)$ & .000 \\
\hline Social activities & $0.6(0.2)$ & $0.5(0.2)$ & .000 \\
\hline \multicolumn{4}{|l|}{ Patient illness severity } \\
\hline Thinking disorder & $6.2(5.4)$ & $7.9(6.0)$ & .000 \\
\hline Withdrawal-retardation & $6.7(5.3)$ & $7.8(5.9)$ & .017 \\
\hline Anxious-depressed & $7.0(4.7)$ & $7.2(4.5)$ & .563 \\
\hline Hostile-suspicious & $3.4(3.3)$ & $4.5(3.8)$ & .000 \\
\hline Activation & $3.4(2.8)$ & $2.6(2.7)$ & .006 \\
\hline Medical comorbidity & $2.97(2.8)$ & $2.4(2.7)$ & .028 \\
\hline Alcohol/drug diagnosis* & $21.4 \%$ & $32.9 \%$ & .003 \\
\hline
\end{tabular}

VA, Department of Veterans Affairs; SD, standard deviation; ADLs, activities of daily living; IADLs, instrumental activities of daily living

${ }^{*}$ Because this variable was dichotomous, the values in the table represent the percentage of inpatient and outpatient providers who are female (gender) or who have an alcohol or drug use diagnosis. The statistics are based on a chi-square test of independence.

${ }^{\dagger}$ The degrees of freedom vary with the type of test, $t$ test with equal variances, $t$ test with unequal variance, and chi-square test of independence.

\section{Patient characteristics}

Aggregate patient characteristics were assessed as the averages across patients within units of several separate dimensions of patient functioning and illness severity (see Table 1). Table 1 summarizes the aggregate indicators of patient functioning and illness severity.

Aggregate patient functioning was assessed by unit-level measures of activities of daily living (ADLs), instrumental activities of daily living (IADLs), social activity, and social contact. ADLs ${ }^{35}$ were measured by a six-item scale that assessed ability to care for basic physical needs (ie, eating, 
bathing, grooming, toileting, and so on). IADLs ${ }^{36}$ were measured by an eight-item scale that assessed the ability to look after day-to-day tasks, such as money, diet and medication management, household activities and chores, social activities, errands (eg, the post office, store, mailbox), and being left alone. ADLs and IADLs were rated on a scale of 0 to $3(0=$ "can perform all essential activities," $1=$ "can perform most essential activities," 2 = "cannot perform most essential activities," and $3=$ "cannot perform the activity"). Higher scores on both measures indicated greater impairment and lower functioning.

Patients' levels of social activity were assessed using the 16-item Daily Activities and Functioning scale from the Quality of Life Interview. ${ }^{37-39}$ Patients indicated their participation in 16 social activities (eg, go for a walk outside, go to a movie, eat at a restaurant, play cards). Outpatients and inpatients who had been in the hospital for more than a month were asked to indicate which of these activities they had been involved in during the month prior to the interview, while inpatients who had been in the hospital less than 1 month reported the frequency of these activities during the month prior to their hospitalization. Each item was scored as $1=$ yes and $0=$ no. Higher scores indicated greater social activity.

Aggregate levels of patients' social contact were measured by five items from the six-item Social Relations scale of the Quality of Life Interview (the item asking how often the patient visited with a close friend was omitted). ${ }^{37-39}$ These items assessed frequency of patients' social contacts in person, over the telephone, by letter, in a joint activity, or as time spent with a boyfriend or girlfriend or with other people, not including those with whom they lived. Item scores were: $0=$ "not at all," $1=$ "less than monthly," 2 = "at least once a month," 3 = "at least once a week," and 4 = "at least once a day." Higher scores indicated more social contact.

Aggregate patient illness severity was measured by the severity of psychiatric symptoms, degree of medical comorbidity, and comorbid drug and alcohol use. The Brief Psychiatric Rating Scale (BPRS) assessed the number and severity of psychiatric symptoms. ${ }^{40,41}$ The 19-item BPRS used in this study is rated from $0=$ "not present" to $6=$ "extremely severe," with the total score ranging between 0 and 114. Higher scores indicate greater symptoms. The 19-item BPRS includes items for four subscales that have been repeatedly identified in factor analysis, including "thinking disorder," "withdrawal-retardation," "anxious-depression," and "hostile-suspiciousness." 42

Medical comorbidity and alcohol and drug use were assessed using data from the VA's Austin automation system data archive. Medical comorbidity was calculated for each patient as the total number of unique comorbid medical diagnoses. Alcohol and drug use was measured by a trichotomous indicator: $0=$ "no alcohol or drug comorbidity," $1=$ "either a comorbid alcohol or drug use diagnosis," and 2 = "both a comorbid alcohol AND drug use diagnosis."

\section{Analyses}

The conceptual model for this research encompassed two sets of variables that bridged organizational levels: those measuring individual health care providers and those characterizing the health care context (ie, unit). Hierarchical Linear Modeling (HLM) was employed to test the hypotheses for this study because it is designed to address two problems common to the analysis of multilevel data. First, aggregation bias occurs when individual-level data (ie, tenure) are aggregated to the group level (ie, average provider tenure), thereby ignoring variation at the individual level. Second, group-level effects are underestimated when the non-independence of responses in a nested data structure are ignored (ie, level of provider job satisfaction varies across units but patterns of covariation are common across units). Conventional statistical approaches fail to address these two issues appropriately. resulting in the underestimation of differences among units. ${ }^{43-45}$

Prior to hypothesis testing, fully unconditional one-way analysis of variance (ANOVA) models with random effects were tested. These models assessed whether multilevel modeling was appropriate, given the observed patterns of central tendency, variation, and reliability in the measures. ${ }^{43}$ 
Next, models examining each of the three measures of provider job satisfaction were developed in two steps. First, means as outcomes regression models constructed at level-1 tested the association between provider characteristics and provider job satisfaction. Provider characteristics were added to the model in the following order: staff background characteristics, staff tenure, and staff occupational rank. Second, unit-level indicators (ie, unit characteristics and measures of the unit patient population) were added to the level- 1 means as outcomes regression models to test the association between the patient components of the health care context while accounting for the effects of provider and unit characteristics.

The one-way ANOVA model with random effects took the following form:

$$
\text { Provider job satisfaction }=\gamma_{00}+\mu_{0 j}+r_{i j}
$$

and the means as outcomes regression model took the following form:

$$
\text { Staff job satisfaction }=\gamma_{00}+\gamma_{i 0}(\text { Level-1 predictors })_{j}+\gamma_{01}(\text { Level- } 2 \text { covariate })_{j}+\mu_{0 j}+r_{i j}
$$

where $i$ corresponds to one of the seven level-1 covariates.

\section{Results}

\section{Preliminary analyses}

Table 1 compares providers working on inpatient and outpatient units. Providers in outpatient units had significantly more education, higher occupational rank and shorter position tenure, more global job satisfaction, and more satisfaction with their relationships with patients.

One-way ANOVA models indicated that the maximum likelihood estimates of the grand means for global job satisfaction $\left(\gamma_{00}=5.097\right.$, standard error of mean $\left.[\mathrm{SE}]=0.123\right)$, satisfaction with relationships with patients $\left(\gamma_{00}=5.420, \mathrm{SE}=0.115\right)$, and satisfaction with workload $\left(\gamma_{00}=3.865\right.$, $\mathrm{SE}=0.133)$ were all significantly greater than $0\left(p_{\mathrm{t}}<.000\right)$. The maximum likelihood estimates of the provider-level variance for global job satisfaction, satisfaction with relationships with patients, and satisfaction with workload were $\sigma^{2}=1.984, \sigma^{2}=.768$, and $\sigma^{2}=1.564$, respectively, and the between unit variances for global job satisfaction $\left(\tau_{00}=0.119\right)$, satisfaction with relationships with patients $\left(\tau_{00}=0.173\right)$, and satisfaction with workload $\left(\tau_{00}=0.191\right)$ were significantly greater then zero $\left(p_{\chi}{ }^{2}<.01\right)$, indicating that most of the variance in these three outcomes was at the provider level. The intraclass correlations $\left(\rho=\tau_{00} /\left(\tau_{00}+\sigma^{2}\right)\right)$, which measures the proportion of variance between units were $\rho=0.057$ (global job satisfaction), $\rho=0.183$ (satisfaction with relationships with patients), and $\rho=0.109$ (satisfaction with workload) indicating that only $6 \%, 18 \%$, and $11 \%$ of the variance in global job satisfaction, satisfaction with relationships with patients, and satisfaction with workload was between units. The reliabilities of the sample means for global job satisfaction, satisfaction with relationships with patients, and satisfaction with workload were $\lambda=0.441, \lambda=0.724$, and $\lambda=0.599$, respectively, indicating that the sample means varied substantially across units and were not adequate estimates of the true unit means. These results indicate that multilevel analysis is appropriate and necessary in order to test the hypotheses of this study. ${ }^{46}$

\section{Hypothesis testing}

Due to the small number of hospital units in this study $(n=18)$, it was necessary to test the unit-level variables in separate models. Based on Bonferroni's adjustment for multiple tests (critical value/number of tests) and $\alpha=.05$, adjusted critical values were $p<.025$ for the two models testing unit characteristics (ie, unit type, unit size), $p<.0125$ for the four models testing patient functioning (ie, ADLs, IADLs, social activity, social contact), $p<.01$ for the five models used to test patient 
symptoms (ie, thinking disorder, withdrawal-retardation, anxious depressed, hostile-suspicious, activation), and $p<.025$ for the two models testing comorbidity (ie, medical, alcohol/drug use). Unadjusted post-hoc $p$-values for each unit-level variable are reported below.

\section{Global job satisfaction}

Providers with shorter tenure in their current positions had greater global job satisfaction. In addition, the global job satisfaction of providers was greater on outpatient units $(p=.046)$ and smaller units $(p=.022)$, units in which patient functioning was higher-lower ADLs $(p=.024)$, lower IADLs $(p=.008)$, and more social contact $(p=.003)$-and on units with less severely ill patients-lower scores on thinking disorder $(p=.022)$, withdrawal-retardation $(p=.030)$, and hostile-suspicious $(p=.009)$-and less comorbidity—alcohol/drug use $(p=.028)$. Global job satisfaction also was associated with shorter position tenure.

\section{Satisfaction with relationships with patients as outcome}

Results indicated that satisfaction with relationships with patients was greatest on outpatient units $(p=.034)$ and smaller units $(p=.002)$, units with higher patient functioning-lower ADL $(p<.000)$ and IADL $(p=.001)$, and more social contact $(p<.000)$-and lower patient illness severity-less thinking disorder $(p<.000)$, withdrawal-retardation $(p<.000)$, hostile-suspicious $(p<.000)$-and with lower comorbidity (medical comorbidity, $p=.044$ ). No other unit- or provider-level associations were significant.

\section{Satisfaction with workload as outcome}

No unit- or provider-level characteristics were associated with satisfaction with workload (all $p$-values $>.05$ ).

\section{Discussion}

This study indicates that unit-level patient characteristics are associated with provider job satisfaction. Global job satisfaction and satisfaction with relationships with patients were consistently associated with unit size, patient illness severity, and patient level of functioning. These associations have important implications for policy makers and health care managers supervising the provision of mental and medical services to patients with long-term health care needs.

Unit size and type (ie, inpatient and outpatient units) may contribute to the association between unit characteristics and provider job satisfaction found in this and other studies. Provider job satisfaction may be greater on smaller units due to the greater frequency and type of interaction between providers and health care recipients on these units. ${ }^{3}$ Job satisfaction also is higher among providers who serve patients who show improvement and whose conditions are less severe. ${ }^{1,4,19,21,47}$ Patients with these characteristics are more likely to be outpatients, patients whose conditions are not long term, and patients who are receiving care on smaller units where the patient/provider ratio may be lower.

Associations identified in this study provide insight into unit characteristics that may potentially contribute to provider satisfaction. Providers working on units with higher average patient functioning and on units with less severe patient illness derived greater satisfaction from their jobs than providers working with more difficult-to-treat patient populations (ie, those commonly found on units where patient functioning is lower and illness severity is higher). ${ }^{2}$ Patients receiving care through outpatient services had significantly higher functioning, including greater social activity, social contact, and 
lower ADLs and IADLs than patients on inpatient units. Outpatients' mental illness also was less severe (see Table 1).

However, the patients who are in greatest need of mental health care are those with the most serious mental health conditions, and provider job satisfaction cannot be improved by simply discharging inpatients to outpatient care or by decreasing unit size. Low provider job satisfaction is likely a function of a variety of patient, unit, and organization characteristics. Low provider job satisfaction may reflect key problems in the organization of care, such as a lack of continuity between health care policy and the infrastructure (ie, resources, training, salaries) necessary for providers to turn policy into practice. Hence, the challenge to health care managers and policy makers is to identify measures that will increase the job satisfaction of clinicians who are charged with the mental health care of patients suffering from serious conditions.

\section{Implications for Behavioral Health Services}

One interpretation of the results is that providers feel greater job satisfaction when they are able to be instrumental in improving the lives and health of the patients they serve. Outpatient units and smaller units may be examples of health care contexts that enhance this opportunity. The higher functioning and lower illness severity among outpatients, as well as closer contact between providers and patients on small units, may facilitate patient-provider interaction and the development of rapport, decrease work-related stress and burnout, and increase the job satisfaction of providers. Due to their higher functioning and generally lower illness severity, patients receiving outpatient care are likely to exhibit clear treatment benefits-such as independent living, working, and greater social connection. However, given fiscal constraints, it is unlikely that the number of small units will be increased, and due to the positive contribution of specialization to health care quality and efficiency, increasing patient mix on units is likely to decrease the quality of patient outcomes and increase health care costs.

As current trends in health care continue, small units will be closed, large units will become more numerous, and existing units likely will increase in size. In addition, demands for the specialized and effective treatment of patients with serious conditions will continue to increase. This trend risks worsening the job satisfaction of providers, and it emphasizes the need for the implementation of measures to avoid decreased provider satisfaction and the attrition of skilled, experienced clinicians. Measures that increase provider job satisfaction also will help decrease burnout and turnover and, although it represents only a small portion of total treatment costs, lower rates of turnover will reduce expenditures for the recruitment and training of new staff. While managing provider exposure to demanding patient populations may be one means to improve provider job satisfaction, ${ }^{1,12,48}$ policy makers and managers must provide the infrastructure, treatment facilities, training, and incentives necessary for providers to treat severely, chronically mentally ill patients successfully.

Effective measures for increasing satisfaction may include decreased workloads (patient/ provider ratio), greater schedule flexibility, shorter more intensive work shifts, opportunities for career advancement, increased time for team meetings and team building, increased compensation for additional effort, and rotation across units that vary in patient mix, but require similar specializations. ${ }^{21,47-51}$ Focusing treatment resources on interventions that work in this population, such as family interventions, programs for assertive community treatment (PACT), and newgeneration antipsychotics that have fewer and less severe side-effect profiles also may assist in increasing provider job satisfaction by leading to improved treatment outcomes for patients and increased treatment success for the providers. Finally, selecting providers and managers who have interest and expertise in working with severely ill patients and providing current providers with opportunities to advance their training and improve their competency in treating severe illness will help improve job satisfaction and retention. 


\section{References}

1. Davis MZ. Nursing and the chronically ill patient in the acute-care hospital. Journal of Advanced Nursing. 1984;9(3):257-266.

2. Landeen J, Kirkpatrick H, Woodside H, et al. Factors influencing staff hopefulness in working with people with schizophrenia. Issues in Mental Health Nursing. 1996;17(5):457-467.

3. Lynch SA. Job satisfaction of home health nurses. Home Healthcare Nurse. 1994;12(3):21-28.

4. Repper J, Ford $\mathrm{R}$, Cooke A. How can nurses build trusting relationships with people who have severe and long-term mental health problems? Experiences of case managers and their clients. Jounal of Advanced Nursing. 1994;19:1096-1104.

5. Hays RD. White K. Professional satisfaction and client outcomes: a reanalysis. Medical Care 1987:25(3):259-264.

6. Leveck ML, Jones CB. The nursing practice environment, staff retention, and quality of care. Research in Nursing and Health. $1996 ; 19(4): 331-343$.

7. Weisman CS, Nathanson CA. Professional satisfaction and client outcomes: a comparative organizational analysis. Medical Care. $1985 ; 23(10): 1179-1192$.

8. Weisman CS, Nathanson CA. Professional satisfaction and client outcomes: reply to Hays and White. Medical Care. 1987;25(3):263-264.

9. Chung MC, Corbett J. The burnout of nursing staff working with challenging behaviour clients in hospital-based bungalows and a community unit. International Journal of Nursing Studies. 1998;35(1-2):56-64.

10. Long A, Reid W. An exploration of nurses' attitudes to the nursing care of the suicidal patient in an acute psychiatric ward. Journal of Psychiatric and Mental Health Nursing. 1996;3(1):29-37.

11. Pablo RY. Job satisfaction in a chronic care facility. Dimensions in Health Service. 1976;53(1):36-39.

12. Stewart MJ, Arklie M. Work satisfaction, stressors and support experienced by community health nurses. Canadian Journal of Public Health. 1994;85(3):180-184.

13. Tai TW, Bame SI, Robinson CD. Review of nursing turnover research, 1977-1996. Social Science and Medicine. 1998;47(12):1905-1924.

14. Aiken LH, Smith HL, Lake ET. Lower mortality among a set of hospitals known for good nursing care. Medical Care. 1994;32(8):771-778.

15. Cameron SJ, Horsburgh ME, Armstrong-Stassen M. Job satisfaction, propensity to leave and burnout in RNs and RNAs: a multivariate perspective. Canadian Joumal of Nursing Administration. 1994;7(3):43-64.

16. Goodell TT, Van Ess Coeling H. Outcomes of nurses' job satisfaction. Joumol of Nursing Administration. 1994;24(11):36-41.

17. Jinnett $\mathrm{K}$, Alexander JA. The infuence of organizational context on job satisfaction and quitting intention: an examination of treatment staff in long term mental healthcare settings. Research on Aging. 1999;21(2):176-204.

18. Melchior ME, Bours GJ, Schmitz P, et al. Burnout in psychiatric nursing: a meta-analysis of related variables. Journal of Psychiatric and Mental Health Nursing. 1997;4(3):193-201.

19. Ramirez AJ, Graham J. Richards MA, et al. Mental health of hospital consultants: the effects of stress and satisfaction at work. Lancet. 1996;347:724-728.

20. Anselmi ML, Angerami EL, Gomes EL. Turnover of nursing staff in hospitals of the municipality of Ribeirao Preto. Pan American Journal of Public Health. 1997;2(1):44-50.

21. Blankertz LE, Robinson SE. Turnover intentions of community mental health workers in psychosocial rehabilitation services. Community Mental Health Journal. 1997;33(6):517-529.

22. Buchan J. Counting the cost of stress in nursing. Nursing Standard. 1995;9(16):30.

23. Dean BS, Krenzelok EP. The cost of employee turnover to a regional poison information center. Veterinary \& Human Toxicology. $1994 ; 36(1): 60-61$

24. Jones CB. Staff nurse turnover costs: part II. Measurements and results. Joumal of Nursing Administration. 1990;20(5):27-32.

25. Knapp M. Missiakoulis S. Predicting turnover rates among the staff of English and Welsh old people's homes. Social Science and Medicine. $1983 ; 17(1): 29-36$.

26. Mann EE. A human capital approach to ICU nurse retention. Journal of Nursing Administration. 1989;19(10):8-16.

27. Fortney $\mathrm{J}$, Booth $\mathrm{B}$, Zhang $\mathrm{M}$, et al. Controlling for selection bias in the evaluation of Alcoholics Anonymous as aftercare treatment. Journal of Studies on Alcohol. 1998;59(6):690-697.

28. Ettner SL, Notman EH. How well do ambulatory care groups predict expenditures on mental health and substance abuse patients? Administration \& Folicy in Mental Health. 1997;24(4):339-357.

29. Goldney RD, Elzinga RH, Kent P. Case mix and affective disorders: a comparison of private and public psychiatric systems. Australian \& New Zealand Joumal of Psychiatry. 1996;30(4):511-515.

30. Hermann RC, Ettner SL. Dorwart RA. The infuence of psychiatric disorders on patients' ratings of satisfaction with health care. Medical Care. 1998;3665):720-727.

31. Soderlund N, Milne R, Gray A, et al. Differences in hospital case mix, and the relationship between case mix and hospital costs. Journal of Public Health Medicine. 1995; 17(1):25-32.

32. Stevens VG, Hibbert CL. Edbrooke DL. Evaluation of proposed case mix criteria as a basis for costing patients in the adult general intensive care unit. Anaesthesia. 1998;53(10):944-950.

33. Mezzich JE. Architecture of clinical information and prediction of service utilization and cost. Schizophrenia Bulletin. 1991;17(3):469 474.

34. Alexander JA, Lichtenstein RL, Oh HJ, et al. A causal model of voluntary turnover among nursing personnel in long-term psychiatric settings. Research in Nursing and Health. 1998:21:415-427.

35. Katz S. Assessing self-maintenance: activities of daily living, mobility, and instrumental activities of daily living. Journal of the American Geriatrics Society. 1983:31(12):721-727.

36. Paveza GJ. Cohen D. Hagopian M, et al. A brief assessment tool for determining eligibility and need for community based long-term care services. Behavior, Health and Aging. 1990:1:121-132.

37. Lehman A. Evaluating Quality of Life for Persons with Severe Mental Yllness. Baltimore, MD: The Evaluation Center: 1995.

38. Lehman AF. Measuring quality of life in a reformed health system. Health Affairs, 1995:14(3):90-101.

39. Lehman AF. A quality of life interview for the chronically mentally ill. Evaluation and Program Planning. 1988:11:51-62. 
40. Overall JE, Gorham DR. The brief psychiatric rating scale. Psychology Reports. 1962;10:799-802.

41. Overall JE. The Brief Psychiatric Rating Scale (BPRS): recent developments in ascertainment and scaling. Psychophamacology Bulletin. $1988 ; 24: 97-99$.

42. Hedlund JL, Vieweg BW. The Brief Psychiatric Rating Scale (BPRS): a comprehensive review. Joumal of Operational Psychiatry. $1980 ; 11(1): 48-65$.

43. Bryk AS. Raudenbush SW. Hierarchical Linear Models: Applications and Data Analysis Methods. Newbury Park, CA: Sage; 1992.

44. Lee VE, Bryk AS, A multilevel model of the social distribution of high school achievement. Sociology of Education. 1989:62(3):172-192.

45. Lee VE, Smith JB. Gender equity in teachers' salaries: a multilevel approach. Education, Evaluation and Policy Analysis. $1990 ; 12$ (1):57-81.

46. Bryk AS, Raudenbush SW. Application of hierarchical linear models to assessing change. Psychological Bulletin. 1987;101(1):147-158.

47. Bame SI, Organizational characteristics and administrative strategies associated with staff turnover. Health Care Management Review. $1993 ; 18(4): 70-86$.

48. Schaefer JA, Moos RH. Effects of work stressors and work climate on long-term care staff's job morale and functioning. Research in Nursing and Health. 1996;19:63-73.

49. Blegen MA. Nurses' job satisfaction: a meta-analysis of related variables. Nursing Research. 1993;42(1):36-41.

50. MacRobert M, Schmele JA, Henson R. An analysis of job morale factors of community health nurses who report a low turnover rate. Joumal of Nursing Administration. 1993;23(6):22-28.

51. Packard IS. Motowidio SI. Subjective stress, job satisfaction, and job performance of hospital nurses. Research in Nursing and Health. $1987 ; 10(4): 253-261$. 